\title{
Indicadores para Centros de Referência em Saúde do Trabalhador: proposição de um sistema de acompanhamento de serviços de saúde
}

\author{
Indicators for Occupational Health Reference Centers: \\ proposal of a system for monitoring health services
}

Camila Corrêa Jacques ${ }^{1}$

Bruno Milanez ${ }^{2}$

Rita de Cássia Oliveira da Costa Mattos ${ }^{3}$

'Secretaria de Saúde, Prefeitura Municipal de São Leopoldo. Estrada do Socorro 1440, Arroio da Manteiga. 93000-000 São Leopoldo RS. mila150@terra.com.br

${ }^{2}$ Faculdade de Engenharia, Universidade Federal de Juiz de Fora

${ }^{3}$ Laboratório de toxicologia, Centro de

Estudos da Saúde do

Trabalhador e Ecologia

Humana, Escola Nacional

de Saúde Pública, Fiocruz

\begin{abstract}
This research proposes a framework of indicators to monitor the activities of the Occupational Health Reference Centers (CEREST). CEREST are structural elements of the National Network of Comprehensive Attention to Workers' Health (RENAST) and are coordinated by the General Coordination of Occupational Health of the Ministry of Health (CGSAT). In order to build this framework, we first elaborated a list of indicators based on the norms that govern CEREST's responsibilities. As a strategy to build a participative approach, a Likert scale questionnaire was sent to 173 CEREST coordinators, who were asked to evaluate this preliminary list of indicators. After the return of the questionnaires $(48,6 \%)$, the answers were analyzed, considering the CEREST scope (State or Regional), date of accreditation and location. Indicators with approval rate higher than $75 \%$ were included in the proposed framework. This instrument consisted in 12 indicators for State CEREST and 13 indicators for Regional CEREST. It is expected that the procedures proposed in this research and the framework itself might encourage the government to create a monitoring system for RENAST as well as for health services in general.
\end{abstract}

Key words Occupational health, RENAST, CEREST, Public Policy Analysis, Indicators
Resumo Os Centros de Referência em Saúde do Trabalhador (CEREST) são eixos estruturantes da Rede Nacional de Atenção Integral à Saúde do Trabalhador (RENAST), coordenados pela Coordenação Geral de Saúde do Trabalhador (CGSAT) do Ministério da Saúde. O instrumento apresentado no artigo visa contribuir para o acompanhamento e a futura avaliação da implantação e implementação da RENAST. Com o objetivo de construir um sistema de indicadores para monitoramento dos CEREST, elaborou-se uma primeira listagem de indicadores com base nas determinações previstas pela Portaria GM/MS no 2.437/2005. A listagem foi enviada a 173 coordenadores dos CEREST para avaliação, com base em uma escala do tipo Likert. Retornaram 48,6\% das avaliações, cuja análise foi realizada globalmente e segundo a abrangência, a data de habilitação e a localização dos CEREST. Consideraram-se aprovados os indicadores com $75 \%$ ou mais de aceitação, resultando um instrumento final com 12 indicadores para os CEREST estaduais e 13 indicadores para os CEREST regionais. A descrição dos procedimentos para a construção do instrumento e sua formatação final poderá ser útil no estímulo à construção de indicadores de monitoramento/avaliação de serviços de saúde em geral.

Palavras-chave Saúde do trabalhador, RENAST, CEREST, Avaliação de políticas públicas, Indicadores 


\section{Introdução}

As mudanças sociais e políticas ocorridas no Brasil a partir das décadas de 1980 e 1990, associadas ao contexto da Reforma Sanitária, foram determinantes nas propostas de reformulação das políticas de saúde. Com a Constituição Federal de $1988^{1}$ e a Lei Orgânica da Saúde ${ }^{2}$, a Saúde do Trabalhador se inscreveu definitivamente como política de saúde.

A construção do campo da Saúde do Trabalhador é uma alternativa à prática da Medicina do Trabalho e da Saúde Ocupacional ${ }^{3,4}$, pois, entre outros aspectos, tem como referência principal a figura do trabalhador como polo central e sujeito ativo no processo saúde/doença. A Saúde do Trabalhador, de acordo com o Ministério da Saúde ${ }^{5}$, tem por objetivos a promoção e a proteção da saúde dos trabalhadores, compreendendo procedimentos de diagnóstico, tratamento e reabilitação de forma integrada no Sistema Único de Saúde (SUS).

Em setembro de 2002, foi criada a Rede Nacional de Atenção Integral à Saúde do Trabalhador (RENAST) através da Portaria do Ministério da Saúde de $n^{\circ} 1.679^{6}$. Constitui-se como uma rede de abrangência nacional e como ferramenta estratégica para a disseminação dos princípios e práticas do campo da Saúde do Trabalhador no SUS, em todos os níveis de atenção.

Na estruturação da RENAST, os Centros de Referência em Saúde do Trabalhador (CEREST) ocupam lugar de destaque. Cabe a estes o papel de pólos irradiadores da cultura da produção social das doenças e da centralidade do trabalho nesse processo, provendo suporte técnico e informações, viabilizando ações de vigilância, facilitando processos de capacitação para técnicos, para o controle social e para toda a rede do SUS, além de executar, organizar e estruturar a assistência de Média e Alta Complexidade relacionada com os problemas e os agravos à saúde relacionados com o trabalho.

A Portaria de criação da RENAST previu a implantação de 130 CEREST entre 2002 e 2004, sendo 27 estaduais e 103 regionais, com dotação orçamentária própria. Para tanto, ela incorporou serviços pré-existentes, os chamados Programas de Saúde do Trabalhador (PST), que já dispunham de um acúmulo de conhecimentos e de práticas ${ }^{7}$. Em dezembro de 2005, foi publicada a Portaria GM/MS no $2.437^{8}$ de ampliação da RENAST prevendo a habilitação de novos CEREST (que passariam para 200) e definindo suas atribuições.
Com a referida Portaria consolida-se a proposta de que esses Centros se constituam em ferramentas na disseminação das práticas em Saúde do Trabalhador no SUS, assumindo o papel de suporte técnico e científico junto aos profissionais de todos os serviços da rede e na formação de recursos humanos ${ }^{9-11}$. Tal proposta também se expressa na 27a resolução da $3^{a}$ Conferência Nacional de Saúde do Trabalhador (3a CNST) $)^{12}$, realizada em 2005, e no texto da Portaria GM/ MS no $2.728 / 2009^{13}$. Esta, porém, não contemplou a descrição das funções dos CEREST como as anteriores, prevendo que o Ministério da Saúde elaboraria diretrizes que norteariam as funções e as atribuições desses Centros em documento separado.

Enquanto inscritos no Sistema Único de Saúde, os Centros de Referência em Saúde do Trabalhador compartilham o desafio de garantir a viabilidade e a sustentabilidade social, política e econômica do SUS. Para tanto, precisam demonstrar seu desempenho na atenção à população, 0 que pode ser feito a partir de um sistema de avaliação $0^{14}$, conforme recomendação aos serviços de saúde, de modo geral, pela Organização Mundial da Saúde e pela Organização Panamericana de Saúde. Um sistema de avaliação pressupõe o conhecimento e o monitoramento dos processos e resultados, além da disponibilidade de informações confiáveis, atualizadas e pertinentes.

Hartz ${ }^{15}$ distingue o monitoramento da avaliação, embora lembre suas inter-relações. Para o autor, o monitoramento dos serviços de saúde está voltado para a análise continuada dos sistemas de informação, incentiva a produção e a qualificação dos serviços e o acompanhamento dos processos e resultados. Portanto, o monitoramento é a condição a priori da avaliação e esta última, segundo Braga Junior' ${ }^{9}$, implica em emissão de juízo de valor.

Tanto o monitoramento como a avaliação pressupõem a obtenção de informações ${ }^{16}$. Uma das maneiras de obtê-las é através de indicadores. É possível entender os indicadores como "medidas-síntese" que contêm informações relevantes sobre determinados atributos e sobre o desempenho do sistema de saúde ${ }^{17}$. Servem para o acompanhamento contínuo de ações e intervenções.

Apesar do relato do uso de indicadores desde algum tempo, sua utilização ganhou corpo científico em meados dos anos $1960^{18}$. A partir da década de 1980, o emprego de indicadores cresceu entre gestores e tomadores de decisão devido à necessidade de planejamento para a formulação e a implementação das políticas públicas. 
Desde então, tem-se constatado um uso crescente, transformando-os em uma ferramenta estratégica para o estabelecimento de políticas e prioridades. $\mathrm{O}$ uso de indicadores para o monitoramento contínuo das ações, atrelado a sistemas de informação, é estratégico para a institucionalização do acompanhamento sistemático dos serviços de saúde ${ }^{19}$.

Um indicador é constituído por um conjunto de dados que informam sobre determinado fenômeno, tornando-se uma fonte de informação. Para Jannuzzi ${ }^{18}$, a principal diferença entre dados coletados e indicadores construídos a partir deles é o conteúdo informativo presente no indicador, ou seja, o valor contextual da informação presente. Os indicadores representam muito mais do que um conjunto de dados, pois adicionam valores aos mesmos, convertendo-os em informações a serem usadas pelos tomadores de decisão. A escolha do indicador depende do que vai ser avaliado e de quem utilizará os resultados para a tomada de decisões.

Todas estas considerações fundamentaram a proposta de criação de um instrumento de monitoramento dos CEREST. Parte-se do pressuposto de que as informações obtidas podem auxiliar nos processos de implantação de políticas públicas e de que, a partir do monitoramento e posterior avaliação, é possível redirecionar rumos, corrigir desvios, reforçar aspectos positivos e subsidiar projetos futuros. Tal posicionamento é reforçado por autores como Machado e Santos ${ }^{20}$ que enfatizam a necessidade da criação de indicadores a fim de acompanhar a execução dos custos orçamentários e a efetividade das ações desenvolvidas pelos CEREST.

Com base em tais justificativas propõe-se a questão: quais indicadores podem constituir um instrumento de monitoramento dos CEREST estaduais e regionais? Pretendeu-se, então, a proposição de um instrumento que poderá ser usado pela Coordenação Geral de Saúde do Trabalhador (CGSAT) do Ministério da Saúde para acompanhamento dos CEREST no território nacional, portanto, com indicadores nacionais.

Acompanhamento e avaliação de serviços de saúde são preocupações recorrentes de todos aqueles atores sociais envolvidos nos temas da saúde pública, em especial, os gestores. Acreditase que o instrumento proposto possa ser aplicável a outros serviços ou possa estimular o desenvolvimento de estudos e pesquisas sobre instrumentalização de acompanhamento/avaliação de serviços de saúde.

\section{Método}

A pesquisa foi dividida em quatro etapas: a exploratória, a do trabalho de campo, a de análise e tratamento do material e a de devolução dos dados. A fase exploratória incluiu a definição do objeto, dos objetivos, das questões norteadoras, da amostra e da listagem preliminar de indicadores, bem como a consulta e a elaboração do material teórico.

Para a construção de indicadores foi feita a leitura, o levantamento e a classificação por temas das funções dos CEREST previstas pela Portaria GM/MS no 2.437/2005 ${ }^{8}$. Apesar de a Portaria GM/MS no $2728 / 2009^{13}$ transferir o detalhamento das funções para outro documento, optou-se pelo uso da Portaria já existente de $2005^{8}$ visto que tal detalhamento tinha origem em uma Portaria de $2002^{6}$, que já era de amplo conhecimento e uso pela Rede.

Identificaram-se alguns conjuntos temáticos nas 23 funções descritas para os CEREST estaduais e nas 26 para os CEREST regionais: ações de educação/capacitação, pesquisas e convênios, ações de articulação intra e intersetorial, ações de assistência e ações de vigilância e de organização da rede para as notificações. Os conjuntos temáticos serviram como referência para a consulta a fontes como a Rede Interagencial de Informações para a Saúde (RIPSA), a Organização Panamericana de Saúde (OPAS), o Departamento de Informática do Ministério da Saúde (DATASUS) e a Coordenação de Aperfeiçoamento de Pessoal de Nível Superior (CAPES) que fundamentaram a elaboração de um instrumento preliminar com 18 indicadores: 16 referentes às atribuições dos CEREST e dois relacionados aos recursos provenientes do Ministério da Saúde, discussão corrente junto à RENAST.

Concomitantemente, foram organizados os principais critérios para a seleção de indicadores identificados pela Rede Carmem do Ministério da Saúde ${ }^{21}$, pela Organização Panamericana de Saúde ${ }^{22}$ e por Jannuzzi ${ }^{18}$. Com base nesta consulta, foram escolhidos os critérios que deveriam ser usados na avaliação dos indicadores: validade (se o indicador mede o que se propõe a medir e se é sensível a mudanças e específico ao tema), confiabilidade (se os dados usados pelo indicador podem ser checados e verificados posteriormente), mensurabilidade (se o indicador é quantitativo e os dados fáceis de se conseguir), comunicabilidade (se é compreensível e se possui o mesmo significado para diferentes pessoas) e re- 
levância (se é relevante para a agenda política de saúde e, no caso, se é relevante para a RENAST).

A seguir, foi construída uma escala tipo Likert, modelo criado em 1932 para avaliar atitudes e hoje amplamente empregado em avaliações diversas, caracterizando-se pela apresentação de afirmações aos sujeitos participantes que correspondem a categorias numéricas. Neste trabalho, em afirmações positivas, o resultado de 5 foi estabelecido para 'aprovo totalmente', o resultado de 4 como 'aprovo' e assim por diante na escala utilizada, até o resultado 1 para o 'desaprovo totalmente'. Nas afirmações negativas se inverteu o sistema de correção. A proposta de inclusão de itens positivos e negativos teve o objetivo de evitar que os respondentes se posicionassem mais à direita ou à esquerda da escala automaticamente, sem uma análise mais reflexiva das questões propostas.

$\mathrm{O}$ instrumento preliminar com os 18 indicadores, os critérios de avaliação e a escala, juntamente com um texto de abertura explicativo e instruções de preenchimento, foi montado em uma ferramenta de formulários via internet, o formSUS. Este é um serviço de uso público, desenvolvido pelo DATASUS que possibilita a coleta e a análise de dados. Constituiu-se como um questionário autoaplicativo ao qual se acrescentaram duas perguntas em aberto: uma acerca da opinião sobre o instrumento e uma segunda com sugestões de indicadores.

O instrumento, após avaliação de especialistas, foi enviado, por via eletrônica, aos coordenadores de todos os CEREST habilitados até dezembro de 2008 (173 Centros, sendo 26 estaduais e 147 regionais), juntamente com a justificativa do estudo e o termo de consentimento livre e esclarecido. Tal etapa, acrescida do recolhimento, constituiu a fase de trabalho de campo da pesquisa. Ao se propor um modelo participativo na elaboração do instrumento final se procurou resgatar o caráter relacional do processo de construção de instrumentos de monitoramento/avaliação, assinalado por autores como Noronha ${ }^{23}$ e Pedroza ${ }^{24}$.

Até o encerramento do prazo estipulado para os CEREST se manifestarem, obteve-se 84 formulários preenchidos, que constituíram a amostra trabalhada na análise dos dados. Esta foi dividida em três partes: a primeira, sobre a caracterização e a participação dos respondentes, incluindo as respostas à primeira pergunta aberta; a segunda, com a análise do instrumento propriamente dito por blocos/temas e as respostas da segunda pergunta aberta; e, uma terceira parte que consistiu na elaboração do instrumento final.
Para a análise dos dados, empregaram-se os programas estatísticos do Excel (97-2003) e do SPSS (versão 15.0 e 17.0). A plataforma do formSUS utilizada na elaboração e na aplicação do instrumento preliminar permitiu a migração dos dados para a plataforma Excel. Medidas estatísticas de significância (qui-quadrado), considerando o agrupamento das respostas segundo a abrangência dos CEREST (estaduais ou regionais), a habilitação (habilitados pela Portaria GM/ MS no 1.679/2002 ou pela Portaria GM/MS no 2.437/2005) e a região onde se localizam (CEREST das regiões sul e sudeste - macro sul - ou das regiões norte, nordeste e centro-oeste - macro norte), foram empregadas com base no programa SPSS. Tal procedimento teve o propósito de levantar a necessidade de indicadores diferenciados conforme tais agrupamentos.

Ao se obter dados quantitativos de base probabilística foi possível tecer um panorama geral e obter um conhecimento das tendências e padrões em cada um dos agrupamentos propostos. As perguntas em aberto forneceram dados que demonstram, em parte, as tendências e os padrões constatados. Tal composição permitiu enriquecer a análise e as considerações acerca do instrumento final proposto.

$\mathrm{Na}$ análise, as respostas da escala Likert foram agrupadas em: indiferente (graduação 3), desaprovo (graduações 5 e 4), aprovo (graduações 1 e 2) e não se aplica/não respondeu. Com relação à avaliação dos indicadores pelos coordenadores dos CEREST, utilizou-se o seguinte critério: indicadores com aprovação abaixo de $70 \%$ foram descartados, indicadores com aprovação entre 70 e $75 \%$ foram objeto de reflexão e indicadores com aprovação superior a $75 \%$ foram incluídos na versão final. A partir do processo analítico, procedeu-se a última etapa, qual seja, a construção do instrumento de monitoramento dos CEREST com os indicadores melhor avaliados, sua proposição à CGSAT do Ministério da Saúde e a devolução aos participantes como forma de retorno às suas contribuições.

\section{Resultados e discussão}

O universo da pesquisa foram os 173 CEREST existentes no período de abrangência proposto. A Figura 1 expõe as proporções do universo e da amostra e seus respectivos agrupamentos.

A primeira coluna mostra o percentual de respondentes (84) em relação ao universo (173), correspondendo a $48,6 \%$. Tal percentual foi con- 
siderado satisfatório visto as dificuldades para a coleta dos dados, as quais expressam a difícil articulação entre as instâncias federal, estadual e municipal na gestão do SUS. Constatou-se, também, uma maior participação dos coordenadores dos CEREST estaduais e os da região macronorte, que conta com um maior número de habilitados pela Portaria GM/MS no 2.437/2005.

Dos 84 CEREST que participaram do estudo, 70 responderam à questão aberta com a opinião sobre o instrumento de maneira geral positiva (67\% dos respondentes). Os comentários negativos se referiram, principalmente, à complexidade do instrumento.

A Figura 2 apresenta a avaliação, pelos coordenadores dos CEREST, dos indicadores propostos, onde se constata uma boa aceitação já que nenhum teve menos de $60 \%$ de aprovação.

A seguir, apresenta-se uma análise segundo cada um dos temas e seus respectivos indicadores.

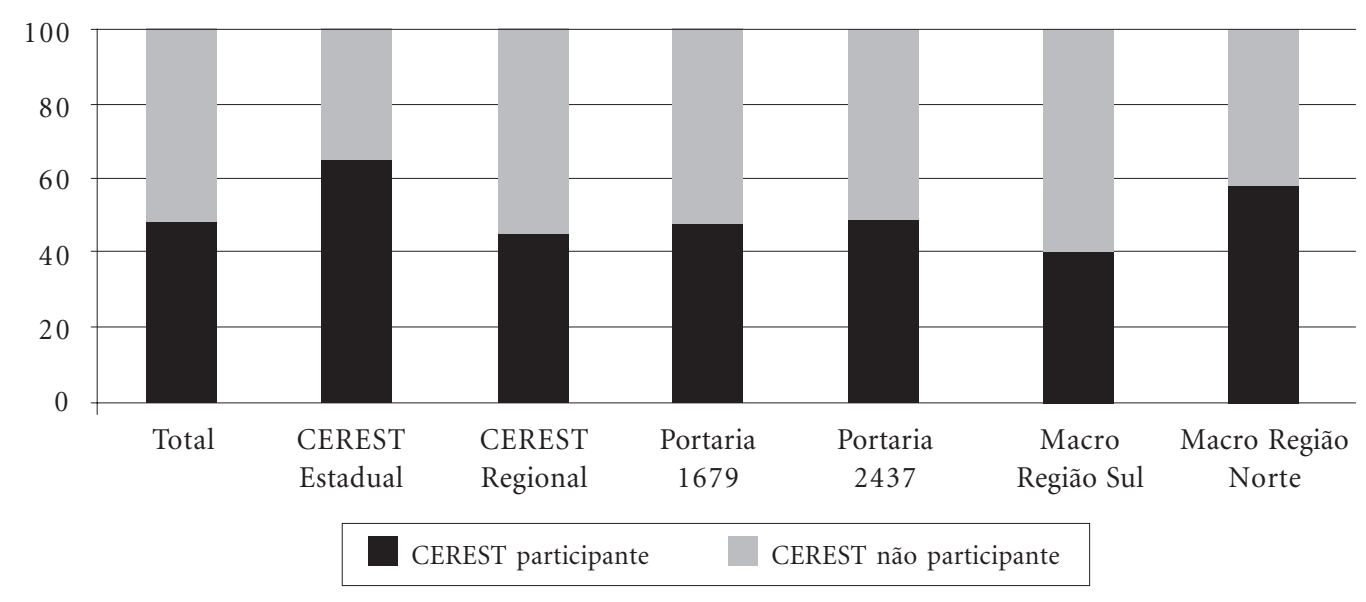

Figura 1. Caracterização da amostra em proporção ao universo segundo a abrangência, a habilitação e a localização.

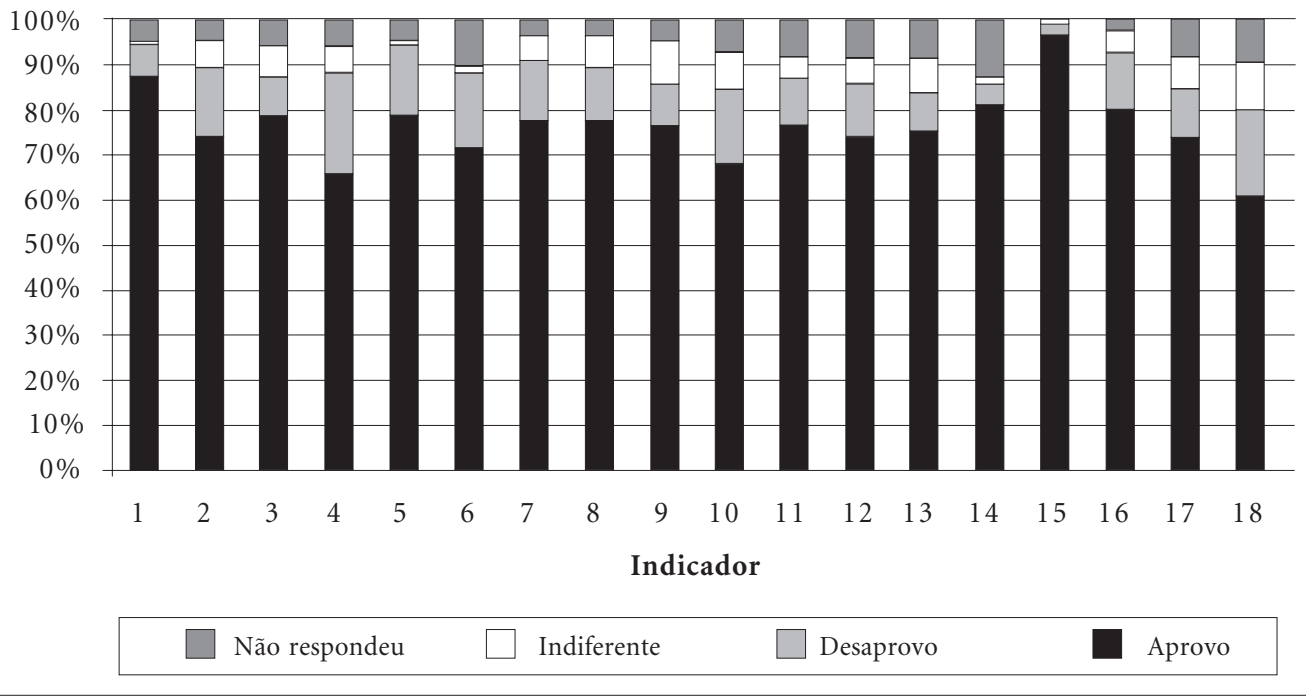

Figura 2. Percentual dos resultados das avaliações dos indicadores pelos CEREST $(n=84)$. 
Compõem o tema 1 (notificação e sistemas de informação) os indicadores 1 (número de notificações de agravos relacionados ao trabalho realizadas pelo CEREST), 2 e 3 (participação dos CEREST no total de notificações no SINAN de acidentes de trabalho e de doenças relacionadas ao trabalho, respectivamente) e 4 (relação entre notificações do SINAN e emissão de CAT). Tais indicadores foram propostos com o objetivo de avaliar o papel dos CEREST nas notificações no Sistema Nacional de Informação de Agravos de Notificação (SINAN) e de relacioná-los com as Comunicações de Acidentes de Trabalho (CATs) do Ministério da Previdência Social. Foram adaptados de indicadores da OPAS/RIPSA, com exceção do indicador 4 . A observação da Figura 2 revela menor aceitação dos indicadores 2 e 4 , com $15,5 \%$ e $22,6 \%$ de desaprovação, o que talvez expresse a dificuldade dos CEREST em contatar com acidentes de trabalho, em geral atendidos em outras unidades de saúde do SUS.

Os indicadores 5 (número de trabalhadores cobertos pelas ações de vigilância realizadas pelos CEREST) e 6 (percentual das ações de vigilância descentralizadas) compõem o tema 2, referente a ações de vigilância empreendidas pelos CEREST. Foram indicadores adaptados do DATASUS referentes às inspeções sanitárias em estabelecimentos. Obtiveram 78,6\% e 71,4\% de aprovação, respectivamente.

No tema 3, relativo à assistência, foram propostos quatro indicadores, fundamentados em indicadores do DATASUS e da OPAS/RIPSA: indicador 7 (número de atendimentos realizados pelos CEREST), 8 (número de relações causais com o trabalho estabelecidas pelos CEREST), 9 (percentual de relações causais em relação ao total de consultas realizadas) e 10 (abrangência geográfica dos atendimentos realizados). Optouse pela inclusão de indicadores de assistência embora este seja um tema controverso na RENAST, pois se julga importante identificar quantos CEREST realizam ações de assistência. Os indicadores tiveram aprovação de 77,4\%, 77,4\%, $76,2 \%$ e $67,9 \%$, respectivamente. O indicador 10 , pior avaliado, inclui ações fora do município sede do CEREST, o que determina dificuldades burocráticas e administrativas e que provavelmente explica a rejeição de $16,7 \%$.

Compõem o tema 4 , referente à articulação interinstitucional e ao controle social, os indicadores 11 (adaptado do DATASUS e que pontua parcerias em ações de vigilância), 12 (número de atendimentos feitos no CEREST referenciados pela rede do SUS em relação ao total de atendi- mentos do CEREST) e 13 (número de reuniões do Conselho de Saúde com pauta sobre Saúde do Trabalhador). Esses tiveram 76,2\% (indicador 11 ), 73,8\% (indicador 12) e 75\% (indicador 13) de aceitação.

Os indicadores 14 (atualização profissional dos trabalhadores do CEREST), 15 (número de ações educativas) e 16 (abrangência das ações educativas realizadas) compõem o tema 5, sobre pesquisa e educação. Estes indicadores têm como referência as Portarias de criação e ampliação da RENAST que apontam como principal missão dos CEREST serem polos irradiadores de conhecimentos para os profissionais da rede do SUS. Eles foram adaptados da OPAS/RIPSA (indicador 14) e da CAPES (indicadores 15 e 16) e obtiveram $81,0 \%, 96,5 \%$ e $79,8 \%$ de aprovação, com destaque para o indicador 15 , aquele com maior aceitação no instrumento.

No último tema (recursos financeiros), foram propostos dois indicadores, adaptados da OPAS/RIPSA: indicador 17 (relação entre o total de recursos financeiros utilizados e o total de recursos recebidos pelos CEREST) e o indicador 18 (relação entre recursos financeiros utilizados em recursos humanos sobre o total de recursos utilizados pelo CEREST). O tema foi proposto diante das frequentes discussões na RENAST acerca dos recursos financeiros disponibilizados. A controvérsia em relação ao tema se expressa na avaliação dos indicadores: $73,8 \%$ de aprovação para o indicador 17 e 60,7\% para o indicador 18 (com 19,1\% de desaprovação), sendo o indicador com menor aprovação no instrumento.

Em síntese, considerando os resultados de forma global, alcançaram aprovação onze indicadores $(1,3,5,7,8,9,11,13,14,15$ e 16) e desaprovação três (4, 10 e 18). Os dois primeiros desaprovados se referem à relação na notificação no SINAN/CAT e à abrangência da área geográfica da assistência prestada pelos CEREST. Estes aspectos são de difícil operacionalização, pois os acidentes de trabalho nem sempre são notificados por CEREST, mas por outros serviços de saúde e as CATs se referem somente a trabalhadores formais; os atendimentos fora do município sede são mais difíceis por questões administrativas e de locomoção. O indicador 18, também reprovado, refere-se ao pagamento dos recursos humanos com verba dos próprios CEREST, tema controverso na Rede.

Os indicadores sugeridos pelos coordenadores dos CEREST para serem agregados ao instrumento, segunda pergunta aberta do instrumento, foram muito variados, sem prevalência. 
Algumas das sugestões poderão se constituir em bons indicadores no futuro. A maioria, no entanto, embora relevante, é de difícil operacionalização no momento e não se dispõe de informações que permitam a avaliação de sua aplicabilidade no atual contexto.

Os indicadores 2, 6, 12 e 17, com índices de aprovação entre $71 \%$ e 74\%, foram objetos de análises com base nas categorias de classificação da amostra. Na categoria abrangência (estadual e regional), o teste de significância apontou um $\mathrm{p}<0,05$ para os indicadores 2 e 10, indicando diferença significativa. Na categoria habilitação e área geográfica, não foram encontradas diferenças significativas entre os segmentos da amostra. O indicador 10 já havia sido excluído (com o significativo índice de $16,7 \%$ de desaprovação) e o 2, que ficou para uma análise mais apurada, foi mantido apenas para os CEREST regionais, visto o significativo percentual de aprovação entre estes.

O indicador 6, sobre a abrangência das ações de vigilância (71,4\% de aprovação), reflete as dificuldades de realizar essas ações em ambientes de trabalho, objeto de discussões recorrentes acerca da competência e da legalidade das mesmas. Optou-se por excluir tal indicador, por ter sido considerado mais oportuno quantificar essas operações quando houver uma posição mais consensual sobre o assunto.

O indicador 12, que não alcançou os percentuais de aprovação, buscou verificar a relação dos CEREST com a rede do SUS através da identificação da origem dos atendimentos. Julgou-se inoportuno sua proposição neste momento porque, em muitos casos, a rede de saúde, incluindo a do trabalhador, ainda está sendo estruturada.

Com relação ao último tema do instrumento, recursos financeiros, o indicador 18 foi excluído. Resolveu-se manter o 17, pois além da questão ser recorrente na RENAST, o monitoramento da utilização dos recursos federais é função do Ministério da Saúde e não se tem como abdicar deste encargo.

Com base nestes resultados, construiu-se o instrumento final de acompanhamento dos CEREST composto de 13 indicadores, mostrado no Quadro 1.

\section{Considerações finais}

O instrumento final apresenta 13 indicadores, sendo que um deles só para os CEREST regionais. Vale ressaltar que os indicadores são construídos com base em informações e dados gerados nos serviços e que não se teve a pretensão de incluir todas as ações que são executadas pelos CEREST, visto sua amplitude.

A exclusão do detalhamento das funções dos CEREST no corpo da Portaria GM/MS no 2.728/ $2009^{13}$ enseja algumas reflexões. Apresenta aspectos positivos, como a flexibilidade para a proposição de ações em acordo com as demandas e estruturas de saúde locais, por outro lado, tal ausência dificultará a proposta de ações pelo desconhecimento e pela pouca clareza sobre o papel dos CEREST. Nesse sentido, a construção e a utilização de um instrumento, com indicadores nacionais, poderão servir como elementos norteadores para a proposição de ações pelos CEREST.

Os indicadores propostos no instrumento final representam um primeiro passo para uma avaliação da RENAST. O instrumento proposto permite que cada Centro avalie seu funcionamento em comparação aos demais, com todas as vantagens que a autoavaliação institucional acarreta. Serve, também, de estímulo para a geração e o armazenamento de informações pelos e entre os CEREST, o que se faz necessário e importante para a construção de indicadores.

O modelo participativo para a construção do instrumento na sua versão final, através da submissão à avaliação pelos coordenadores dos serviços sobre os indicadores propostos, qualificou o instrumento e poderá estimular a participação futura no processo de monitoramento/avaliação dos CEREST. É uma dificuldade recorrente na Rede do SUS a avaliação pelos gestores e pelas instâncias institucionais de formulação e implementação das políticas de saúde dos serviços prestados. Neste sentido, pretende-se, com o detalhamento dos procedimentos empregados e a apresentação do instrumento na sua versão final, estimular a proposição de ferramentas de acompanhamento/monitoramento dos serviços de saúde de modo geral. Para finalizar, reafirmase que, especificamente em relação a este estudo, as informações contribuirão para a avaliação da implementação da Política Nacional de Saúde do Trabalhador. 
Quadro 1. Instrumento de acompanhamento/avaliação dos CEREST:

\begin{tabular}{|c|c|}
\hline \multicolumn{2}{|c|}{ Tema 1 - Notificações e sistemas de informações } \\
\hline $\begin{array}{l}\text { Indicador } 1 \text { - Número de notificações de } \\
\text { agravos relacionados ao trabalho } \\
\text { realizados pelo CEREST. }\end{array}$ & $\begin{array}{l}\text { Método de cálculo: Total das notificações de agravos } \\
\text { relacionados ao trabalho realizados pelo CEREST. } \\
\text { Conceito: Considere como agravos relacionados ao trabalho } \\
\text { aqueles listados na Portaria GM/MS 777/04 (acidentes e } \\
\text { doenças). }\end{array}$ \\
\hline $\begin{array}{l}\text { Indicador } 2 \text { - Participação do CEREST no } \\
\text { total das notificações de acidentes de } \\
\text { trabalho no SINAN (só para os CEREST } \\
\text { Regionais). }\end{array}$ & $\begin{array}{l}\text { Método de cálculo: Total de notificações de acidentes de } \\
\text { trabalho realizadas pelo CEREST (regional) sobre o total de } \\
\text { notificações de acidentes de trabalho nos municípios ou } \\
\text { estado de abrangência do CEREST (X 100). } \\
\text { Conceito: Considere como acidente de trabalho de } \\
\text { notificação compulsória aqueles listados na Portaria GM/MS } \\
777 / 04 \text {. }\end{array}$ \\
\hline $\begin{array}{l}\text { Indicador } 3 \text { - Participação do CEREST no } \\
\text { total de notificações de doenças } \\
\text { relacionadas ao trabalho no SINAN. }\end{array}$ & $\begin{array}{l}\text { Método de cálculo: Total de notificações de doenças } \\
\text { relacionadas ao trabalho realizadas pelo CEREST (regional } \\
\text { ou estadual) sobre o total de notificações de doenças } \\
\text { relacionadas ao trabalho nos municípios ou estado de } \\
\text { abrangência do CEREST (X 100). } \\
\text { Conceito: Considere como doenças relacionadas ao trabalho } \\
\text { aquelas listadas na Portaria GM/MS 777/04. }\end{array}$ \\
\hline \multicolumn{2}{|l|}{ Tema 2: Vigilância } \\
\hline $\begin{array}{l}\text { Indicador } 4 \text { - Número de trabalhadores } \\
\text { cobertos pelas ações de vigilância } \\
\text { realizadas pelo CEREST. }\end{array}$ & $\begin{array}{l}\text { Método de cálculo: Total de trabalhadores das empresas } \\
\text { onde foram realizadas ações de vigilância pelos técnicos do } \\
\text { CEREST. } \\
\text { Conceito: Considere como ações de vigilância as visitas aos } \\
\text { ambientes de trabalho. }\end{array}$ \\
\hline \multicolumn{2}{|l|}{ Tema 3 - Assistência } \\
\hline $\begin{array}{l}\text { Indicador } 5 \text { - Número de atendimentos } \\
\text { realizados pelo CEREST. }\end{array}$ & $\begin{array}{l}\text { Método de cálculo: Número total dos atendimentos } \\
\text { realizados pelo CEREST. } \\
\text { Conceito: Considere como atendimento as consultas } \\
\text { (médicos, enfermeiros, fisioterapeutas, etc), os } \\
\text { procedimentos e as atividades de acolhimento. }\end{array}$ \\
\hline $\begin{array}{l}\text { Indicador } 6 \text { - Número de relações causais } \\
\text { com o trabalho estabelecidos pelo } \\
\text { CEREST. }\end{array}$ & $\begin{array}{l}\text { Método de cálculo: Número total de relações causais entre } \\
\text { agravo (doença/acidente) e o trabalho estabelecidos pelo } \\
\text { CEREST. } \\
\text { Conceito: Considere relação causal a relação entre o agravo } \\
\text { (consequência) e o trabalho (causa). }\end{array}$ \\
\hline $\begin{array}{l}\text { Indicador } 7 \text { - Percentual de relações } \\
\text { causais estabelecidas pelo CEREST em } \\
\text { relação ao total de consultas realizadas no } \\
\text { CEREST. }\end{array}$ & $\begin{array}{l}\text { Método de cálculo: Total de relações causais entre agravo } \\
\text { (acidente e doença) e trabalho estabelecidas pelo CEREST } \\
\text { sobre o total de consultas realizadas pelo CEREST (X 100). } \\
\text { Conceito: Considere relação causal a relação entre o agravo } \\
\text { (consequência) e o trabalho (causa). }\end{array}$ \\
\hline
\end{tabular}




\begin{tabular}{|c|c|}
\hline \multicolumn{2}{|c|}{ Tema 4 - Articulação interinstitucional e controle social } \\
\hline $\begin{array}{l}\text { Indicador } 8 \text { - Parcerias em ações de } \\
\text { vigilância. }\end{array}$ & $\begin{array}{l}\text { Método de cálculo: Total de ações em parceria de vigilância } \\
\text { sobre o total de ações de vigilância realizadas somente pelo } \\
\text { CEREST (X 100). } \\
\text { Conceito: Considere como ações de vigilância em parceria } \\
\text { as visitas aos ambientes de trabalho realizadas pelos técnicos } \\
\text { do CEREST em conjunto com outras entidades públicas que } \\
\text { não da área da saúde, tais como o Ministério Público do } \\
\text { Trabalho, Ministério do Trabalho e outros. }\end{array}$ \\
\hline $\begin{array}{l}\text { Indicador } 9 \text { - Número de reuniões do } \\
\text { Conselho de Saúde com pauta sobre } \\
\text { Saúde do Trabalhador. }\end{array}$ & $\begin{array}{l}\text { Método de cálculo: Número de reuniões do Conselho de } \\
\text { Saúde onde a Saúde do Trabalhador foi ponto de pauta sobre } \\
\text { o total de reuniões do Conselho. } \\
\text { Conceito: Os CEREST devem acompanhar a pauta do } \\
\text { Conselho Estadual de Saúde; enquanto que os CEREST } \\
\text { regionais a pautas do Conselho Municipal de Saúde, do seu } \\
\text { município sede. }\end{array}$ \\
\hline \multicolumn{2}{|l|}{ Tema 5 - Pesquisa e educação } \\
\hline $\begin{array}{l}\text { Indicador 10: Atualização profissional dos } \\
\text { trabalhadores do CEREST. }\end{array}$ & $\begin{array}{l}\text { Método de cálculo: Número de postos de trabalho do } \\
\text { CEREST que possuem pós-graduação sobre o número total } \\
\text { de postos de trabalho do CEREST (X 100). } \\
\text { Conceito: Considere como curso de pós-graduação } \\
\text { aperfeiçoamento, especialização, mestrado e doutorado em } \\
\text { saúde pública/coletiva ou saúde do trabalhador. }\end{array}$ \\
\hline $\begin{array}{l}\text { Indicador 11: Número de ações } \\
\text { educativas. }\end{array}$ & $\begin{array}{l}\text { Método de cálculo: Total das ações educativas realizadas pelo } \\
\text { CEREST para não trabalhadores do CEREST. } \\
\text { Conceito: Considere ações educativas cursos, palestras, } \\
\text { oficinas e seminários. }\end{array}$ \\
\hline $\begin{array}{l}\text { Indicador } 12 \text { - Abrangência das ações } \\
\text { educativas realizadas pelo CEREST. }\end{array}$ & $\begin{array}{l}\text { Método de cálculo: Somatório do produto do número de } \\
\text { alunos pelo total de horas das ações educativas realizadas } \\
\text { para não-trabalhadores do CEREST (número de alunos do } \\
\text { curso } 1 \text { X carga horária do curso } 1 \text { ) }+ \text { (número de alunos do } \\
\text { curso } 2 \text { X carga horária do curso } 2 \text { ) + .... } \\
\text { Conceito: Considere ações educativas cursos, palestras, } \\
\text { oficinas e seminários. }\end{array}$ \\
\hline \multicolumn{2}{|l|}{ Tema 6 - Recursos financeiros } \\
\hline $\begin{array}{l}\text { Indicador } 13 \text { - Relação entre o total de } \\
\text { recursos financeiros utilizados e o total de } \\
\text { recursos recebidos pelo CEREST. }\end{array}$ & $\begin{array}{l}\text { Método de cálculo: Recursos financeiros utilizados pelo } \\
\text { CEREST sobre o total de recursos recebidos. } \\
\text { Conceito: Considere apenas os recursos referentes à Portaria } \\
\text { GM/MS 2437/05, fornecidos pelo Ministério da Saúde. }\end{array}$ \\
\hline
\end{tabular}

\section{Colaboradores}

CC Jacques escreveu este artigo a partir de sua dissertação de mestrado; B Milanez e RCOC Matos foram os orientadores. 


\section{Referências}

1. Brasil. Constituição da República Federativa do Brasil. 17a ed. São Paulo (SP): Saraiva; 1997.

2. Brasil. Lei no 8.080, de 19 de setembro de $1990-e$ legislação Correlata. 2a ed. Porto Alegre (RS): Corag; 2002.

3. Nardi H. Saúde do Trabalhador. In: Cattani AD, organizador. Trabalho e tecnologia; dicionário crítico. Petrópolis: Vozes; 1997. p. 219-224.

4. Lacaz FAC. O campo da Saúde do Trabalhador: resgatando conhecimentos e práticas sobre as relações trabalho-saúde. Cad Saude Publica 2007; 23(4):757-766.

5. Brasil. Ministério da Saúde (MS). Doenças Relacionadas ao Trabalho: manual de procedimentos para os serviços de saúde. Brasília: Ministério da Saúde (MS); 2001.

6. Brasil. Ministério da Saúde (MS). Portaria GM/MS no 1.679 , de 19 de setembro de 2002 - Dispõe sobre a estruturação da Rede Nacional de Atenção Integral à Saúde do Trabalhador no SUS. Diário Oficial da União 2002; 20 set.

7. Vasconcellos, LCF de. Saúde e trabalho e desenvolvimento sustentável: apontamentos para uma política de Estado [tese]. Rio de Janeiro: Fiocruz; 2007.

8. Brasil. Ministério da Saúde (MS). Portaria GM/MS no 2.437, de 07 de dezembro de 2005. Dispõe sobre a ampliação e o fortalecimento da Rede Nacional de Atenção Integral à Saúde do Trabalhador - RENAST no Sistema Único de Saúde e dá outras providências. Diário Oficial da União 2005; 8 dez.

9. Braga Júnior D. Gestão da Rede Nacional de Atenção Integral à Saúde do Trabalhador - RENAST. In: $3^{a}$ Conferência Nacional de Saúde do Trabalhador - Coletânea de textos. Brasília; Ministério da Saúde (MS); 2005. p. 83-90.

10. Braga Júnior D. Rede Nacional de Atenção Integral à Saúde do Trabalhador - Manual de Gestão e Gerenciamento. São Paulo: Hemeroteca Sindical Brasileira; 2006.

11. Dias E, Hoefel MG. O desafio de implementar as ações de saúde do trabalhador no SUS: a estratégia da RENAST. Cien Saude Colet 2005; 10(4):817-828.

12. Brasil. Ministério da Saúde (MS). Resoluções da $3 a$ Conferência Nacional de Saúde do Trabalhador. 2006. [acessado 2008 set 14]. [Documento da Internet]. Disponível em: http://bvsms.saude.gov.br/bvs/ trabalhador/pdf/relatorio_final_3con.pdf

13. Brasil. Ministério da Saúde (MS). Portaria GM/MS no 2.728, de 11 de novembro de 2009. Dispõe sobre a Rede Nacional de Atenção Integral à Saúde do Trabalhador (RENAST) e dá outras providências. Diário Oficial da União 2009; 12 nov.
14. Viacava F, Almeida C, Caetana R, Fausto M, Macinko J, Martins M. Uma metodologia de avaliação do desempenho do sistema de saúde brasileiro. Cien Saude Colet 2004; 9(3):711-724.

15. Hartz ZM. Pesquisa em avaliação da atenção básica: a necessária complementação do monitoramento. Divulgação saúde para debate 2000; 21:29-35.

16. Novaes HMD. Avaliação de programas, serviços e tecnologias em saúde. Rev Saude Publica 2000; 34(5):54-59.

17. Organização Panamericana de Saúde (OPAS). Indicadores básicos para a saúde no Brasil: conceitos e aplicações. Brasília: Organização Panamericana de Saúde (OPAS); 2008

18. Jannuzzi PM. Indicadores sociais no Brasil: conceito, fontes de dados e aplicações. Campinas: Alínea; 2006.

19. Santos-Filho SB. Perspectivas da avaliação na Polí tica Nacional de Humanização em Saúde: aspectos conceituais e metodológicos. Cien Saude Colet 2007; 12(4):999-1010.

20. Machado JMH, Santos APL. O financiamento na indução de ações em Saúde do Trabalhador: o caso da Rede Nacional de Atenção Integral à Saúde do Trabalhador. No prelo 2010.

21. Brasil. Ministério da Saúde (MS). Guia metodológico de avaliação e definição de indicadores crônicos não transmissiveis e Rede Carmem. Brasília: Ministério da Saúde (MS); 2007.

22. Organização Panamericana de Saúde (OPAS). Indicadores básicos para a saúde no Brasil: conceitos e aplicações. Brasília: Organização Panamericana de Saúde (OPAS); 2008.

23. Noronha O. Pesquisa participante: repondo questões teórico-metodológicas. In: Fazenda I, organizadora. Metodologia da pesquisa educacional. São Paulo: Cortez; 1989. p. 137-143.

24. Pedrosa JI. Perspectivas na avaliação em promoção da saúde: uma abordagem institucional. Cien Saude Colet 2004; 9(3):617-626.

Artigo apresentado em 06/07/2010

Aprovado em 20/11/2010

Versão final apresentada em 10/12/2010 